\title{
471896 - GABAPENTIN PHARMACOKINETICS AND FEASIBILITY IN CARDIAC SURGERY
}

\author{
Joel Parlow, MD FRCPC, Brian Milne, MD, Ian Gilron, MD, Debbie Shore, RN, \\ Elizabeth Orr, RN \\ Anesthesiology, Kingston General Hospital, Kingston, ON, Canada
}

Introduction: Gabapentin (Gbp) has been shown to decrease postoperative pain and opioid requirements, and to improve postoperative pulmonary function(1). Its use in the setting of cardiac surgery has not been reported to date. We carried out a pilot study to examine Gbp pharmacokinetics during and after cardiopulmonary bypass as well as the feasibility of its use as an analgesic adjunct for pain following cardiac surgery.

Methods: Following ethics approval and informed consent, 16 patients undergoing primary elective CABG, with planned fast track recovery (anticipated extubation 1-4 hours postoperatively), were recruited to this open-label observational study. Patients received oral Gbp $600 \mathrm{mg}$ preoperatively, repeated following tracheal extubation and every eight hours until the evening of the first postoperative day (POD1). Gbp levels, analyzed using HPLC separation and mass spectometry detection,were measured at induction, prior to $\mathrm{CPB}, 10$ min after $\mathrm{CPB}$ commenced, just prior to separation from $\mathrm{CPB}, 30$ min following $\mathrm{CPB}$, and prior to and $2 \mathrm{~h}$ following the next 3 doses of gabapentin. Perioperative hemodynamics, and postoperative pain scores during rest, sitting, coughing and forced expiration were documented using visual analog scale. Total opioid use, sedation scores and side effects were recorded.

Results: Gbp blood concentrations remained stable during and following CPB. Fourteen patients completed the protocol, and were extubated between $15 \mathrm{~min}$ and $4.5 \mathrm{~h}$ postoperatively. Gbp levels remained stable over the next 24h, but accumulated by the end of POD1 (Figure, mean \pm SD). Total morphine use in ICU was $13 \pm 10 \mathrm{mg}$ (mean \pm SD). Pain scores were similar to those in the post-sternotomy setting in previous studies.

Discussion: At the time of writing, this is the first reported study examining the use of Gbp in cardiac surgery. Cardiopulmonary bypass did not affect Gbp concentration, but drug levels accumulated on POD1 using this regimen. This pilot study shows the feasibility of using Gbp in the cardiac surgical population, and will enable future controlled studies examining safety and analgesic efficacy.

References: (1)Pain 2005; 113:191-200. 


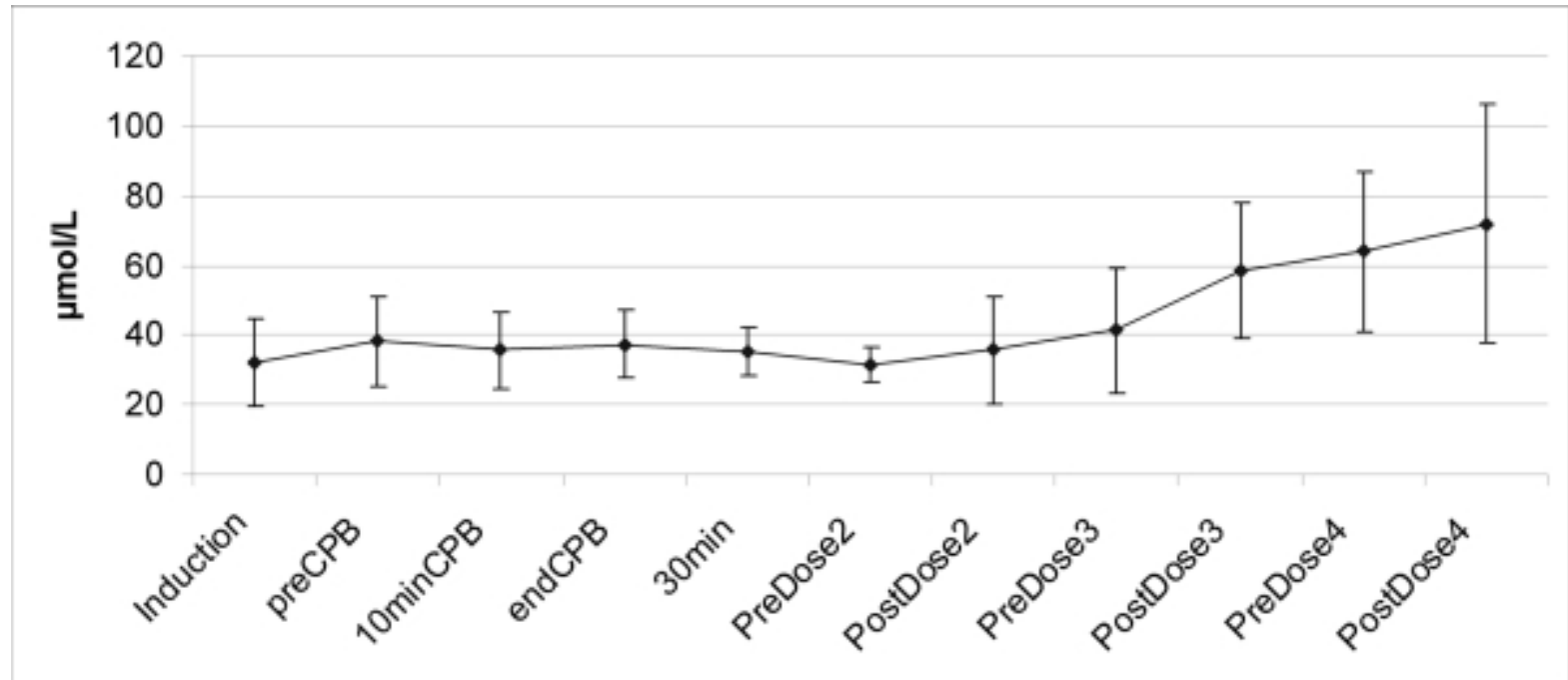

Gabapentin concentrations before, during and after cardiopulmonary bypass (mean,SD) 